\title{
AntarcticAR: An Outdoor AR Experience of a Virtual Tour to Antarctica
}

\author{
Gun A. Lee $^{1 *}$ Andreas Dünser $^{2} \quad$ Alaeddin Nassani $^{1}$ Mark Billinghurst $^{1}$ \\ The Human Interface Technology Laboratory New Zealand (HIT Lab NZ), University of Canterbury ${ }^{1}$ \\ CSIRO Computational Informatics $(\mathrm{CCl})^{2}$
}

\begin{abstract}
In this paper we introduce AntarcticAR, a mobile outdoor Augmented Reality (AR) application that provides a virtual tour of Antarctica. Outdoor AR interfaces have often been used as a navigational tool, providing information related to the real world environment where the user is located in. In contrast, this research explores using outdoor AR to provide a virtual tour that has little relationship with the user's real environment. The AntarcticAR application allows people to virtually visit places of interest in Antarctica or follow historic expeditions to the South Pole. The paper describes the design and implementation of the application, and reports on a user study with members of the public. Users felt that the application provided a compelling virtual tour, and that the outdoor AR technology especially enriched the experience.
\end{abstract}

Keywords: Outdoor augmented reality, virtual tour, mobile application, location-based system.

Index Terms: H.5.1 [Information Interfaces and Presentation]: Multimedia Information Systems - Artificial, augmented, and virtual realities

\section{INTRODUCTION}

Virtual tours have been one of the main applications of Virtual Reality (VR) technology. For instance, the Sensorama was an early immersive multi-sensory experience that allowed people to take a virtual bicycle ride through Brooklyn [17]. By allowing users to travel within a simulated environment, a virtual tour gives an opportunity to visit places that are remote in space and time.

Visiting new places is not only an entertaining experience, but also allows people to discover new facts, gain knowledge, and better understand the nature of the world they are traveling in. The knowledge gained from having firsthand experience is not comparable to that obtained from indirect experiences (e.g. reading books), hence many museums attempt to recreate remote environments through their exhibits. However, physically replicating remote places is costly and not always possible.

Immersive VR has been one of the main ways to deliver virtual tour experiences. By presenting computer simulated virtual environments, VR systems allow users to navigate and interact within a virtual space from a first person perspective. Creating three-dimensional (3D) models for a virtual tour is often easier than building a physical replica of the touring environment. Another benefit of using VR technology is being able to present different tours using the same VR equipment. However, immersive VR theatres are usually expensive, and users still have to physically visit the facility to have the virtual experience.

* e-mail: gun.lee@canterbury.ac.nz

IEEE International Symposium on Mixed and Augmented Reality 2013 Arts, Media, \& Humanities Proceedings

1 - 4 October 2013, Adelaide, SA, Australia

978-1-4799-2945-0/13/\$31.00 @2013 IEEE
In this research, we investigate using mobile outdoor Augmented Reality (AR) as an alternative. Originally, mobile outdoor AR systems required a significant amount of hardware that the users had to carry [6][7][16]. However, mobile phones and tablets have become powerful enough to run AR applications, and many AR experiences are now available for use in consumer market [25][26][29]. At first, these outdoor AR applications simply showed geo-located information such as icons and text labels overlaid on the real world. But recently, they became capable of visualizing 3D models registered in the real world [10]. While previous mobile outdoor AR applications focused on augmenting real world tours, this research explores using outdoor AR to provide a virtual tour into an environment that has little relationship with the place where the user is currently located.

In this paper we introduce AntarcticAR (see Figure 1), an AR application that provides a virtual tour to Antarctica, one of the most astonishing continents in the world, and a challenging place to visit. Using mobile outdoor AR technology, AntarcticAR brings Antarctica to the place where the user is, so that he or she can personally have an experience of exploring an exotic world.

In the next section we review related work that uses VR and AR technology for touring application. Then we describe the motivation and design goals of this project, followed by the implementation details. We also describe user studies that we have conducted, and use the results to provide design guidelines for developing similar mobile outdoor AR applications.



Figure 1: AntarcticAR application showing a virtual scene of Scott's hut in Antarctica overlaid in the real world.

\section{Related Work}

Traditionally, virtual tours use immersive visualization technology to immerse visitors into a virtual trip. IMAX [23] movie theatres present visually immersive films of exotic venues all over the world, while VR theatres attract visitors into an interactive tour to ancient cities [15] or even imaginative worlds [14]. While these types of systems provide physically immersive experience to the visitors, they are usually not widely accessible. 
As personal computing technology developed, virtual tour applications became available on desktop computers. The introduction of digital panorama pictures [4] made it possible to show remote environments to the user with interactive 360-degree photographs. Now people can watch panorama pictures of streets in many cities all over the world on the web [1]. The development of computer graphics technology also made interactive 3D visualization common on personal computers, especially in games. Today, people not only can explore imaginary virtual worlds in online games, but can also fly over 3D modeled cities around the globe [22]. While these technologies made virtual tours more accessible, desktop virtual tour applications still fall short of providing immersive experiences. Personal immersive display technology (e.g. head-mounted displays (HMD) or 3D projectors) is expected to reduce this problem as it becomes more accessible in the future.

While VR focuses on reproducing touring experience in virtual space, AR tries to enhance tours in the real environment. Outdoor AR technology has focused on tour applications from its earliest development. The Touring Machine [6] is one of the earliest outdoor AR systems, and it provided in-situ information to the user while touring in an outdoor environment. Information provided in this system included text labels, images, and 3D models registered to the real world, web pages and video clips shown on a handheld display, and panoramic pictures with the view turning according to the user's head movement [8]. Showing virtual buildings registered in the real world is a common feature of outdoor AR tour applications [8][19], and recently a smartphone application was developed for showing AR buildings in an earthquake damaged city [10].

Most outdoor AR applications focus on showing information related to the place where the user is located. In comparison, our project focuses on bringing a virtual environment to the user and providing information related to this remote place. This is similar to outdoor AR games [5][12][18] in a sense that both present a virtual scene that has little relationship with the real world. On the other hand, they are different from our project, which focuses on users traveling into the virtual scene, rather than virtual game objects appearing in the real world.

Our application is similar to a dislocative tourism project 'You Are Not Here' [30] where participants use a double side printed map to have a tour of Baghdad through the streets of New York. While this project used a printed map and an audio tour guide provided through a cell phone, we use outdoor AR technology to provide a richer user experience.

In terms of showing an AR scene of a remote place, our system is similar to the CityViewAR application [10], which allows users to choose a virtual viewpoint at the remote place. While the virtual viewpoints in CityViewAR were fixed positions, in our system, users can freely travel around the virtual scene registered to the real environment.

Compared to other AR systems, our project sits on a different position in Milgram's Mixed Reality continuum [13]. While it is an AR application, our system sits closer to the virtual environment side of the continuum because it replaces significant portions of the real world with virtual content. However, it is different from Augmented Virtuality systems where objects in the real environment are used to augment the virtual environment.

Our system works as if it is revealing a virtual space coexisting in the real world, so that the users could travel through and experience this virtual environment while maneuvering in the real environment. In this work we call this type of outdoor AR experience an 'Augmented Parallel Reality', inspired by the concept of parallel universe in many science fiction novels and movies.

\section{ViRTual TOUR to ANTARCtica using OUtDOOR AR}

\subsection{Antarctica - a Perfect Destination for Virtual Tours}

Antarctica is one of the most extraordinary continents in the world. With its coldest temperature ever recorded $\left(-89.2{ }^{\circ} \mathrm{C}\right)$, and averaging 166 millimeters of annual precipitation, Antarctica is the coldest, driest, and windiest place in the world on Earth [20]. In Antarctica, the sun moves along the horizon and never sets for several months, while it never rises in other months of the year, leaving night skies with the Aurora Australis. As 98\% of the continent is covered in ice, ice sheets can be up to 3 kilometers thick, which makes Antarctica the highest continent with average elevation of 2,300 meters. Sea ice, ice bergs, glaciers and crevasses create an exclusive landscape, with distinctive wild animals, including penguins, seals, and whales.

With its extremely unique environment, Antarctica has been one of the most interesting places to visit for many explorers and scientists, albeit being one of the hardest places to get to. How explorers strived with their historic expeditions and what tools and technologies the scientists are using today to discover and live in Antarctica are further stories that makes this unique continent an even more interesting place.

Being one of the most fascinating and difficult places to visit, Antarctica is a perfect destination to visit through a virtual tour. Many museums try to convey this experience through exhibits with pictures, movies, installations, and even immersive theatres [2][24]. There are plenty of things to discover, learn, and enjoy by visiting this exceptional place. Our aim was to allow people to experience Antarctica in any real environment.

\subsection{Design Goals for AR Virtual Tour to Antarctica}

The goal of the project was to develop an AR system that provides an immersive, interactive, and personal virtual tour experience for the public, so that they can discover new facts, gain new knowledge, and enjoy visiting Antarctica.

To provide this unique experience through an AR system, we set four design goals for this project:

1) Immersive: The system should provide immersive visualization of the tour destination. This does not necessarily mean physical immersion, but rather providing a high level of user perceived immersion from a cognitive point of view.

2) Interactive: The system should be responsive to users' input, and users should be able to interactively participate in the experience, rather than passively observe the tour environments.

3) Accessible: The system should be widely accessible to the general public. It should be affordable for personal use, usable by a wide range of age groups, require little physical ability, and be socially acceptable.

4) Compatible: The system should be able to cope with different types of content. Immersive visualization of the virtual tour environment should not prevent users from accessing more conventional types of content, such as pictures and movie clips. Furthermore, it should take advantage of these by weaving them into the virtual touring experience.

After considering different options, we found that an outdoor AR application using handheld mobile devices could meet many of these requirements.

Although mobile devices usually have small screens that are not comparable to immersive VR theatres, Hwang et al. [9] showed that a handheld display with motion tracking can provide a comparable amount of immersion to that provided by larger screens when showing virtual environments. In that sense, outdoor $\mathrm{AR}$ applications on a mobile device have the potential to provide an immersive visualization of a composite environment around the user. With tracking the users' view using GPS and orientation sensors, outdoor AR applications can provide users the experience 
of freely walking in an augmented environment. Physically walking in an environment is a feature that even many immersive VR theatres cannot provide.

Modern mobile devices, such as smartphones and tablets, are widely used. They have an easy and intuitive interface using touch screen and motion sensors, enough processing power to perform everyday tasks and are small and light enough to carry anywhere. All of these features at an affordable price have made them widely adopted by the public and so mobile devices have become one of the most popular AR platforms [10][12][25][26][29].

While HMDs can provide better visual immersion, they do not have such as large user base and the social acceptance of handheld devices. Using handheld devices also makes it easier to support other types of content, such as text, images, and video clips, along with an AR view. Image quality on current handheld devices is also far better than that of most affordable HMDs.

Overall, when considering accessibility and immersiveness, outdoor AR on handheld mobile devices could be a good choice for virtual tour applications. It can provide an accessible and immersive touring experience into a virtual environment with additional information provided through other types of media.

\subsection{AntarcticAR}

Based on the goals and requirements, we designed a mobile application called AntarcticAR that provides an outdoor AR experience of a virtual tour to Antarctica. The virtual tour can take place in any location where users can freely walk around, but we first presented the experience at a local event named NZ IceFest. This is a festival held in the city of Christchurch, celebrating and sharing Antarctica's science, heritage and culture.

As we collected content available for use in the tour, we found that there are many different themes varying from simple facts about the environment in Antarctica to historic stories of expedition to the South Pole. In order to meet different interest of various audiences, we decided to design multiple tours with different themes and let the user choose between them. In this way, we made each tour more focused, while also providing customized experiences to the users based on their preferences.

In the application, we refer to different tours as 'missions'. Each mission consists of a set of locations to visit and a route that the user should follow to complete the mission. By asking the users to complete a mission, they are more encouraged to actively participate in the tour and make progress by visiting each point of interest (POI) included in the mission. We designed four missions with different themes that users can choose (see Table 1). The first two missions involve visiting places and learning about modern Antarctica. The other two missions are designed to match the route and experience of historic expeditions to Antarctica.

Designing an outdoor AR experience requires mapping a virtual world to the real environment. Many outdoor AR applications simply map the virtual objects (e.g. buildings) to the corresponding geographical coordinates in the real world $[8][10][19]$. In comparison, Augmented Parallel Reality applications can have different options since there is no direct spatial relationship between the virtual touring environment and the real world. The mapping can be arbitrary, as in some outdoor AR games [12] that randomly place the virtual objects around the real world location of the user. Alternatively it can be as straight forward as shifting the geographical coordinates to a remote site.

In this project, we shrunk down the continent of Antarctica to fit into a much smaller physical space. Next to the venue of the festival, there was a field on a park that was about 300 meters wide and long, and into this space we mapped the POIs of the virtual tour. Instead of randomly mapping the locations, we wanted the users to have a sense of walking through a scaled model of Antarctica with correct topological structure.
Table 1: Missions for virtual tour to Antarctica

\begin{tabular}{l|c|l}
\hline \multicolumn{1}{c|}{ Title } & Type & \multicolumn{1}{c}{ Description } \\
\hline $\begin{array}{l}\text { Explore } \\
\text { Deep Field }\end{array}$ & Modern & $\begin{array}{l}\text { Visit Dry Valleys, Mt. Erebus, Ross Sea, } \\
\text { Skeleton Glacier, and learn about their } \\
\text { environment and inhabiting creatures. } \\
\text { Visit places around Ross Island and learn } \\
\text { about the people at Scott Base, and the } \\
\text { environment they are living in. } \\
\text { Visit } \\
\text { Ross Island }\end{array}$ \\
$\begin{array}{l}\text { Scott's } \\
\text { Expedition to } \\
\text { the South Pole }\end{array}$ & Historic & $\begin{array}{l}\text { Follow Scott's last expedition to the } \\
\text { South Pole and learn how Scott and his } \\
\text { party traveled through Antarctica. }\end{array}$ \\
$\begin{array}{l}\text { Hillary's Tractor } \\
\text { Drive to } \\
\text { the South Pole }\end{array}$ & Historic & $\begin{array}{l}\text { Learn about Hillary's 1956 expedition } \\
\text { and follow his tractor drive to the South } \\
\text { Pole. }\end{array}$ \\
\hline
\end{tabular}

In order to use the available physical space as much as possible, instead of mapping the entire continent to the field, we decided to map only the region of Antarctica which included the POIs in the tour. Moreover, since different missions had different sets of POIs, the mapping (scale, translation and orientation) between the real and virtual world also varied depending on the mission. Users with different missions still shared the same physical space, so we tried to design the route of the missions not to coincide with each other so that the foot traffic could be distributed over the whole field.

We chose tablet computers as the device for running the application as we focused on designing the application for using at the festival. While smartphones are more widely used, tablets have bigger screens that can show more content and are easier to watch, while still being light enough to carry.

In the next section, we describe the details of the user interface design of the AntarcticAR mobile application.

\section{USER INTERFACE DESIGN}

Figure 2 shows the navigational structure of the AntarcticAR application. This was designed with the user in mind to allow for browsing different types of content during a mission. Using the application, users go through three mission phases while they experience the virtual tour; (1) Mission Start, (2) Mission InProgress, and (3) Mission End.

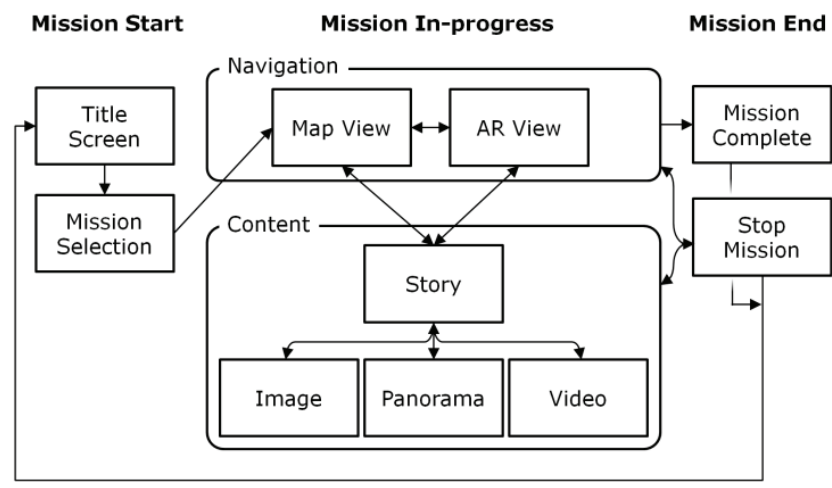

Figure 2: View navigational structure of AntarcticAR application.

Mission Start: At the beginning of the tour, the user is welcomed by a title screen followed by the Mission Selection screen (see Figure 3) allowing the user to read brief descriptions about the missions that they can choose from. 
Mission In-Progress: Once selected, the mission starts with the Map view that shows a map of the part of Antarctica that the user is touring. During the mission, the user can switch between the two navigation views (Map and AR) to see their location and the surrounding area in the virtual tour environment. Using these views, the user can travel to various POIs. When users reach a POI, they will be shown a Story view which allows them to read and view the content of the current location, such as text, images, panoramic views and videos.

Mission End: After the user has visited all of the POIs in the mission and followed the route to the end, they will get a congratulation message. Alternatively, the user can stop the mission while it is in progress. Once the mission has ended, the application will go back to the title screen allowing the user to select a different mission and restart from the beginning.

In the rest of this section we explain the design of each view of the application in more detail.



Figure 3: Mission selection screen.

\subsection{The Map and AR Views}

The Map view (see Figure 4) shows the map of Antarctica where the user is virtually travelling. The virtual tour space is mapped to the real environment where the user is physically located, so that the user travels through the virtual space by moving in the real environment. The position and orientation of the user in the physical space is tracked using the GPS and compass sensors of the mobile device. Based on this tracking information the application updates the user's location and orientation on the map, where it is shown as a blue arrowhead.

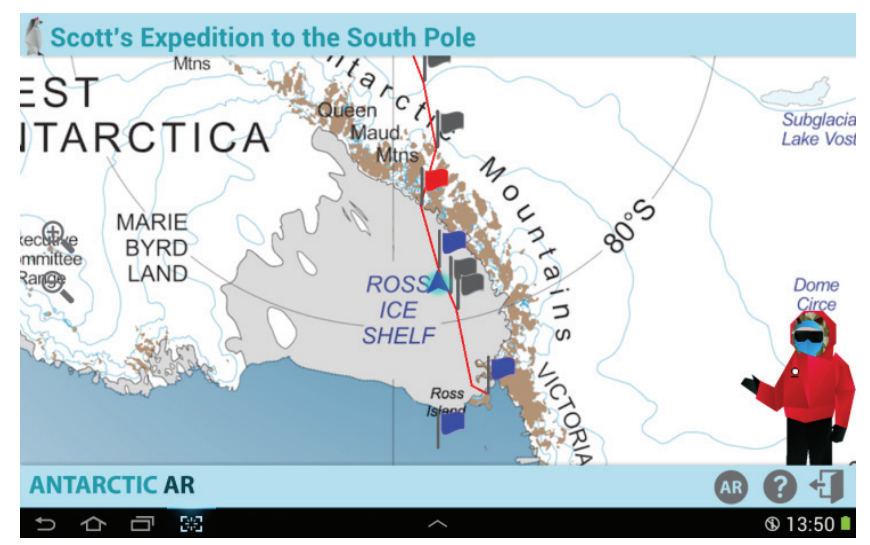

Figure 4: Map view showing POls on a map of Antarctica.
The map shows the places that the user needs to visit as flags connected via a red route path. These are color coded as red, blue and grey. The red flag is the next destination where the user has to travel to, the blue flags are the places already visited, and the grey flags are the places to visit in the future.

Similar to the Map view, the AR view (see Figure 1 and 5) also serves as another navigational tool by displaying 3D models overlaid on top of the real camera view. While the map provides an exocentric view of the world, the AR view gives a first-person view of the virtual tour environment.

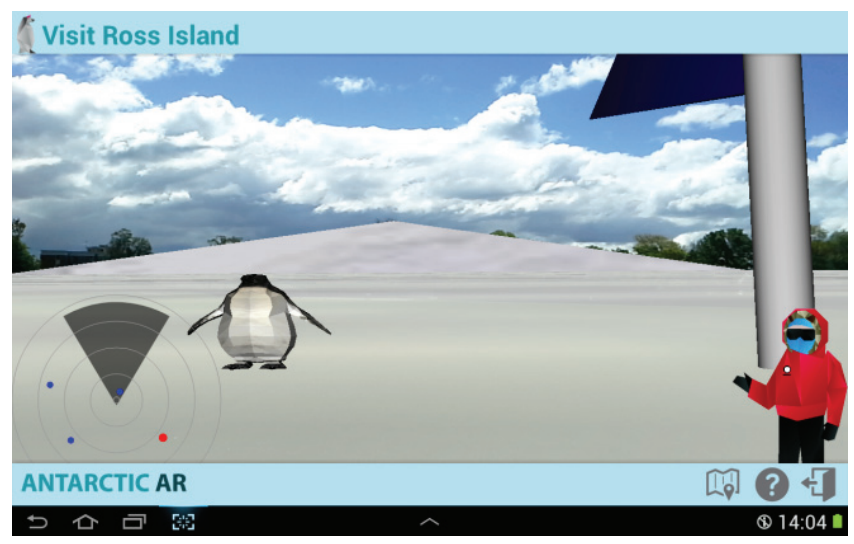

Figure 5: AR view showing a virtual scene overlaid in the real world.

In the AR view, a semi-transparent white surface is overlaid on the real ground to simulate ice and the horizon is bounded by white mountains, creating a visual representation of Antarctica. Through the semi-transparent ice on the ground and above the mountains, the user can see the real world including the sky and the surrounding environment. As the user travels in the real environment, the virtual view is updated according to the user's movement so that it gives the illusion of travelling within the virtual environment. By showing the virtual scene overlaid on the real world, users can feel more like the virtual space exists where the user is, rather than being confined to the screen on the device.

In the AR scene, each POI is represented by a virtual flag. The colors of the flags follow the same color code of the Map view providing visual consistency. In order to help users easily identify the current target location, the red virtual flag is also animated to rotate around itself. The same color coding is used for the dots on the radar at the bottom left corner of the AR view. The radar helps users find POIs around them, even outside the view.

To provide a richer experience in the AR view, we added extra $3 \mathrm{D}$ objects to some of the POIs, including penguins running around a flag pole, a camp of tents, or an old hut. These virtual objects can help users to be more engaged with the virtual world. In order to prevent users getting distracted from the current target POI, these objects are not revealed until the user has reached the corresponding POI on the tour route.

A virtual character is shown in the lower right corner of both the Map and AR views and narrates the story in the mission. The character is a tour guide who greets the user, tells the story, and gives hints on what to do next. The message from the guide character appears in speech bubbles (see Figure 6). In order to not disturb the user, the word bubbles are designed to disappear after a couple of seconds. However, if needed, the user can view the last message again by tapping on the guide character. 


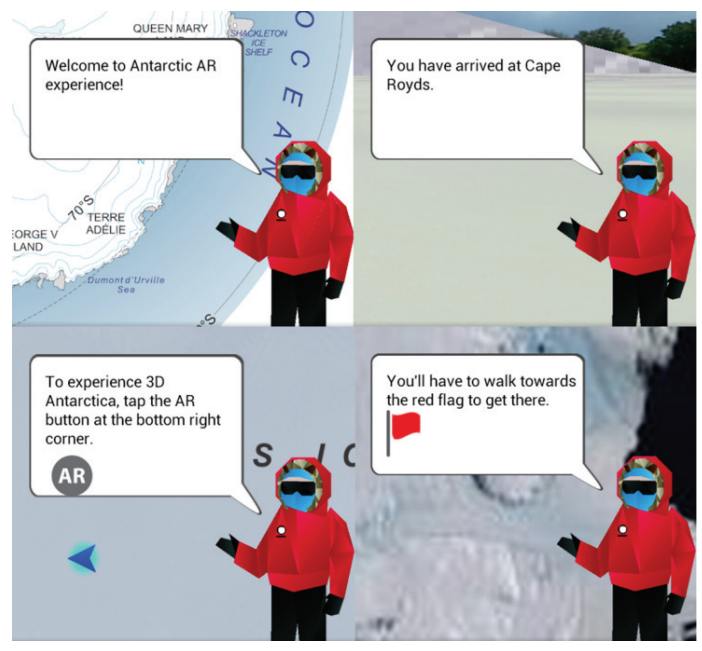

Figure 6: Messages from the virtual guide character.

\subsection{Content Views}

There are four types of content shown in AntarcticAR: Story, Image, Video and Panorama. When the user arrives at the current POI, the Story view appears on the screen describing the place with a few pages of text and thumbnail images (Figure 7). The user can swipe on the touch screen to turn the pages, and tap on a thumbnail image to see the linked pictures, videos, or panoramas. Once the user has finished viewing the content, he or she can close the view by tapping the close button at the top right corner.
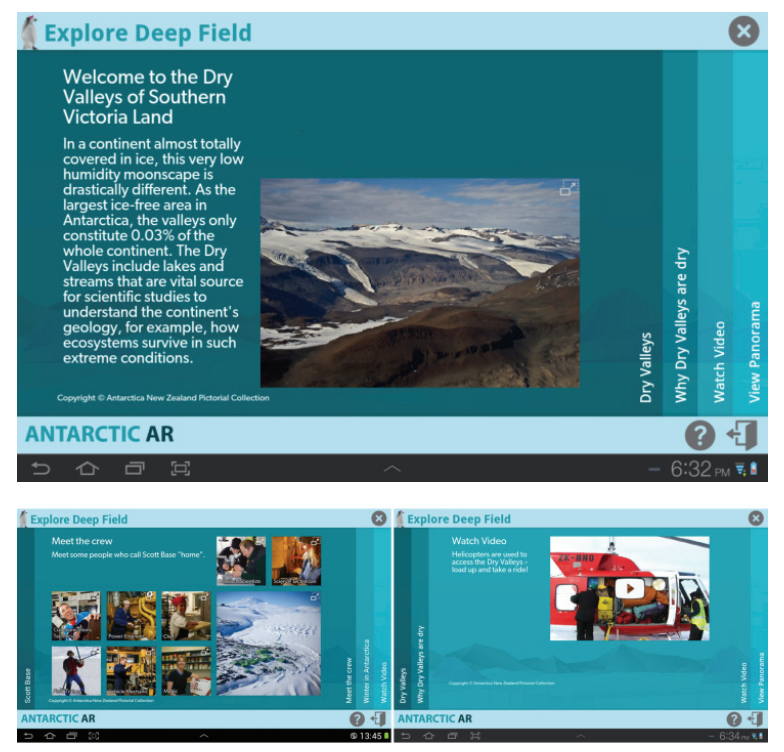

Figure 7: Story pages of the Explore Deep Field mission.

Each type of content has a slightly different user interface. In the Image view (top of Figure 8) users can see a picture full screen and use multi-touch gestures to scroll and zoom the image. The Video view plays a video clip and provides video controls including a play/pause button and a progress slider with which the user can use to control the playing part.

The Panorama view shows 360-degrees panoramic images that are responsive to the orientation sensors; when the user turns the device, the viewing direction of the panorama picture turns accordingly. The user can also stop using the orientation sensor by either tapping on the icon at the top left corner or dragging the panorama picture to turn the view using the touch screen.
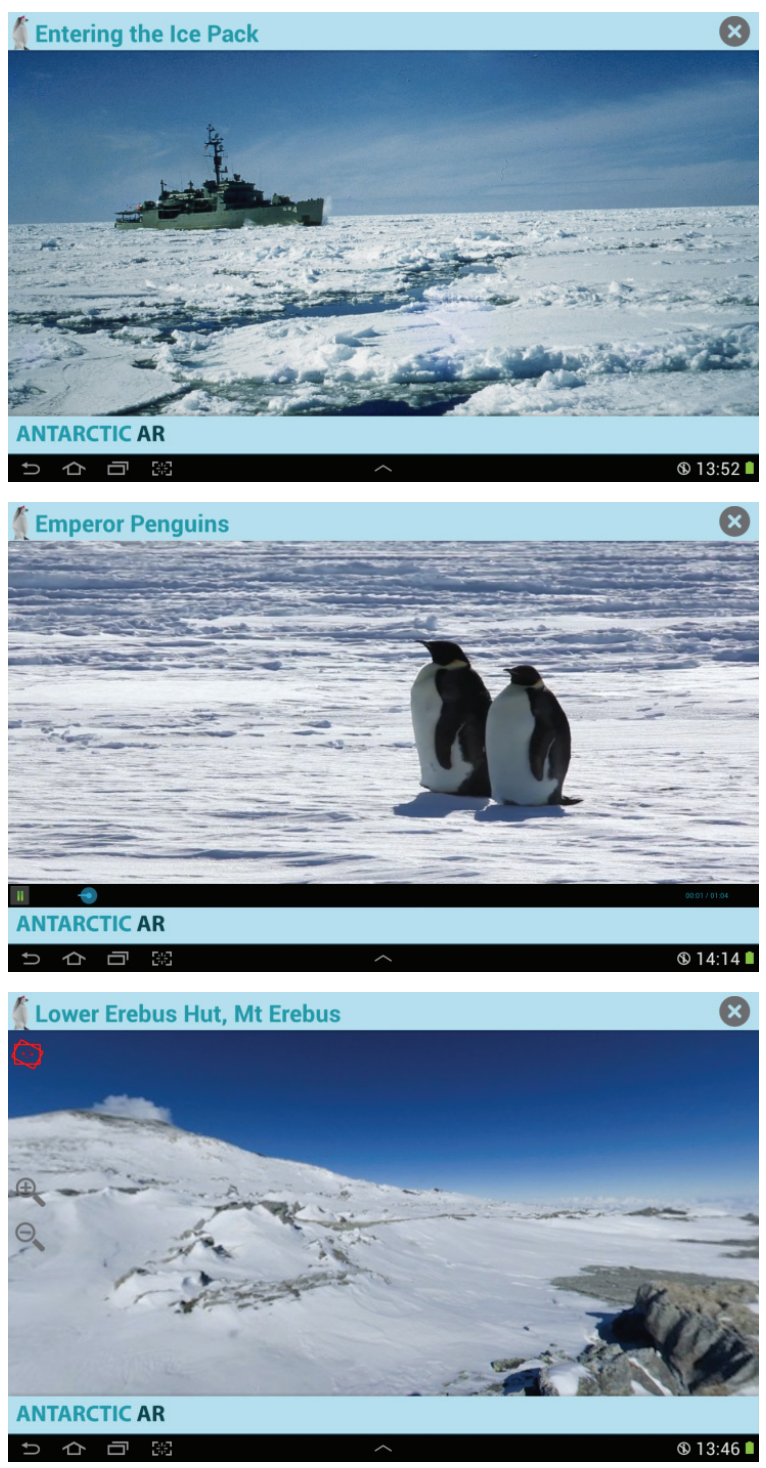

Figure 8: Image, Video and Panorama views (from top to bottom).

\section{IMPLEMENTATION}

The AntarcticAR application was developed on the Google Android platform and it runs on tablet devices running Android OS version 3.1 or higher. We used the Outdoor AR library [27] to get position and orientation information based on the sensors available on the tablet (i.e. GPS, accelerometer, gyroscope, and compass), and the AR view component in the library to show the AR scene with 3D models at a given geo-location.

To draw the map of Antarctica, we developed a custom map view based on the image viewing functions in the Android SDK. This was implemented by creating an interface for scrolling and zooming a large image with a map of Antarctica, and overlaying a set of icons (e.g. user's location and POIs) on the map image.

In the application we wanted to remap the Antarctic continent down to a much smaller physical space that the user could walk through. To map the real geographic coordinates (i.e. latitude and longitude) onto the image coordinates of the map, we used bilinear interpolation based on a set of corresponding pairs of geographical and image coordinates. Although the geographical coordinate space is non-linearly mapped onto a flat surface, considering the range of the geographical coordinate space we are 
using (size of a field on a park), we regarded it as a linear mapping without much problem for implementing the application. While two corresponding coordinate pairs would be enough for uniformly mapping the image space to the geographical space, in order to allow non-uniform mapping between the two spaces, we divided the image space into grids based on the provided coordinate pairs and use bi-linear mapping to each section of the grid. Figure 9 shows examples of how POIs in the real world are mapped to the map image. While the topology of the corresponding POIs on the Antarctic map and the real map remains similar, the relative distance between the POIs are squashed and stretched to make the walking distance between the POIs more evenly distributed.

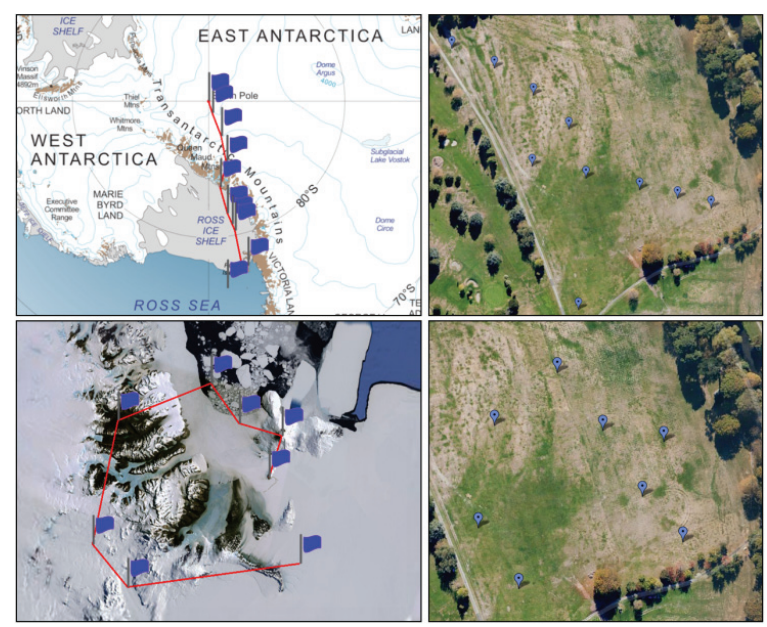

Figure 9: Mapping of image coordinates to geographical points for the Scott's Expedition to the South Pole mission (above) and the Explore Deep Field mission (below).

The tour information is stored in a SQLite database, including a list of POIs for each mission. Each POI has a name, location, associated content and optional 3D models. The content and 3D models are stored as asset files in the application.

The progress through the mission is based on the location of the user. Starting with the first POI in the mission as a target location, when the user gets close enough to the geographical coordinate of the current target location, the content related to that location is shown to the user. When the user finishes viewing the content by closing the view, the next POI in the mission is set as a new target location until there is no more POI left. Every time a new target location is set, the state of the mission is recorded in the local storage so that the application can recover to the last known state after any unintended termination. This helps users to continue their mission from where they left it if the application crashes or they accidentally quit.

While the main user interaction is to walk to the POIs in the outdoor environment, there could be times when this is not possible (e.g. due to bad weather). In such cases, the application has a special mode for indoor use where users can use touch screen interaction instead. To move to the target location, the user simply taps on the flag (either on the map or AR view), and the application will move the user's location close to the POI. In this case, the application uses mock locations instead of the live values from the GPS sensor. By faking the user's location, the map view can show the user icon at the intended position, and the AR view can show the virtual scene from the intended perspective.

The story content is stored and rendered using HTML pages, while images and video uses standard file formats supported by the Android platform (e.g. JPEG or MPEG4). For visualizing the 360-degrees panoramic images we used the PanoramaGL open source library [28]. Each mission has 9 to 10 POIs, and each POI has 2 to 4 pages of stories that have links to pictures, video clips, and panorama pictures. The number of content items depends on the mission, as is summarized in Table 2. The length of each video clip was about a minute on average. In addition to the content, some of the POIs had 3D models related to the story other than flags (e.g. tents, huts, penguins, etc).

Table 2: Amount of content for each mission

\begin{tabular}{c|cccccc}
\hline Mission & POIs & $\begin{array}{c}3 D \\
\text { models }\end{array}$ & $\begin{array}{c}\text { Story } \\
\text { pages }\end{array}$ & Pictures & $\begin{array}{c}\text { Video } \\
\text { clips }\end{array}$ & Panorama \\
\hline Deep Field & 9 & 3 & 34 & 57 & 8 & 2 \\
Ross Is. & 9 & 4 & 19 & 21 & 5 & 3 \\
Scott & 10 & 2 & 21 & 20 & - & 1 \\
Hillary & 9 & 2 & 18 & 39 & - & - \\
\hline
\end{tabular}

\section{USER STUdY}

\subsection{Preliminary Feedback from the Stake Holders}

Prior to the public demonstration of the application, we showed the application to stake holders from the event (five people who were festival organizers and Antarctic scientists). The demonstration was held on the site where the outdoor tour was going to be held. Participants shared a tablet to try the application and gave feedback verbally while discussing how the user experience could be enhanced by improving the design.

The most prominent feedback was to remap the POIs in the real world, so that the traveling time between the POIs would be reduced and more evenly distributed. Initially, we tried to maximize the traveling area in the real world, and maintain the proportion of the distance between the local POIs to match that of the actual places in Antarctica. While this better reflected the relative spatial configuration of the remote environment, it required users to walk a long way between POIs that were placed far apart. There was a discussion to map the POIs in an evenly distributed random shape that had no relation with the actual geography of Antarctica. However, we wanted to at least convey the information of topological structure of the tour route to the users. In the end, we adjusted the distance between POIs to become more evenly distributed, unless the geographical topology is broken or the proportion of the distance between the POIs is meaningful to the story of the mission.

Another feedback was the lack of information about how long the tour was expected to last. Participants suggested it would be better if the users could know how much time was needed to complete the tour, both at the beginning of the mission and during the mission. Based on this feedback, we updated the mission selection screen to provide an estimated running time and walking distance for each mission. To provide the notion of progress during the mission, we chose to modify how the flags on the map are visualized. Besides the red flag, initially the flags for the points already visited were shown in grey and no flags were shown for the future target points. This prevented the screen getting cluttered, but it didn't show how many points were left to visit. The improved design used blue flags for the points already visited and all of the future target points were shown with a grey flag which didn't draw too much attention from the user but was still effective in giving progress cues.

In terms of the user interface, reflections on the screen were a problem. Although adjusting the color contrast improved the visibility of the content slightly, the screen was physically not bright enough to view in an outdoor environment. To overcome this, we added a custom sun visor to the tablet case (see Figure 10). This was made out of card board, folding up into a protective 
case with a sun visor that improved the visibility of the screen outdoors. The top of the sun shade also had printed instructions giving a brief overview about how to use the application.

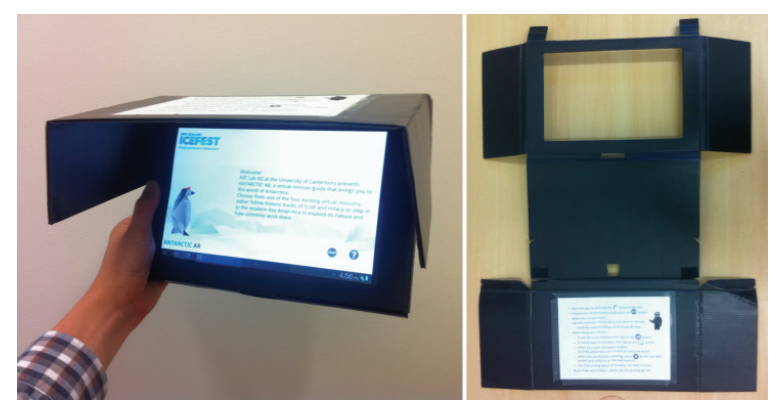

Figure 10: A custom designed protective case with a sun visor.

While most of the touch interactions used in the application were standard and well known to those who have used a tablet before, due to very diverse target audience, no single interaction could be considered straight forward to the user. This was observed during the demonstration to the stake holders, where some had trouble with simple interactions. So we decided to add popup tips that gave hints to the users about how to interact with the application. The tips appeared on the screen for a couple of seconds with an illustration of how to interact with the current screen. They only appeared when the user entered a certain type of screen for the first time, so that they didn't bother the user afterwards. Swiping the pages in the story view, pinching the image to zoom, and rotating the device to change the panorama view are the examples of interactions shown (see Figure 11).

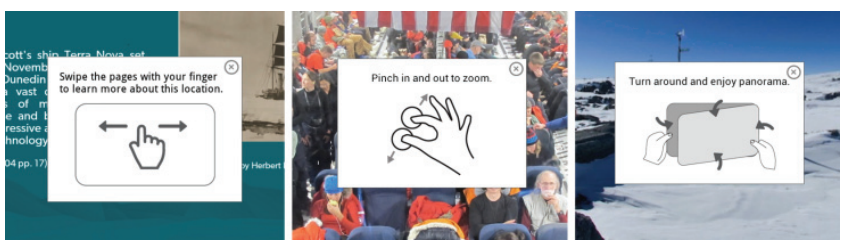

Figure 11: Popup tips in the story, image, and panorama views.

\subsection{Public Demonstration}

We demonstrated the AntarcticAR application to the public at the NZ IceFest festival. During the festival, about 250 people used the application outdoors on one of the fields of North Hagley Park, and over a thousand people tried it indoors. We used eight Samsung Android tablets of various types: Galaxy Note 10.1, Galaxy Tab2 10.1, Galaxy Tab 10.1 and 8.9. Five of them had a screen size of 10.1 inches while three had 8.9 inch screens. Half of them were running Android OS version 4.x while the other half had version 3.x. Depending on the weather or their preference, participants were able to use the application outdoors on the field where the virtual tour was held or in an indoor environment where the demonstration booth was located (see Figure 12).

For those who participated in the outdoor tour, a staff member guided the participants out onto the field, and from there users were able to freely walk around with the tablet. Most of the participants were in a group of 2-7 people, sharing a tablet device between two or three people, while a few participants had a tour alone. Although the participants were mostly on their own during the tour, there was a staff member on the field in order to provide technical support. Each tour lasted for about half an hour depending on the amount of content provided in a mission, the users' interest in the content, and their walking speed. While most of the participants did only one mission, a few did more.

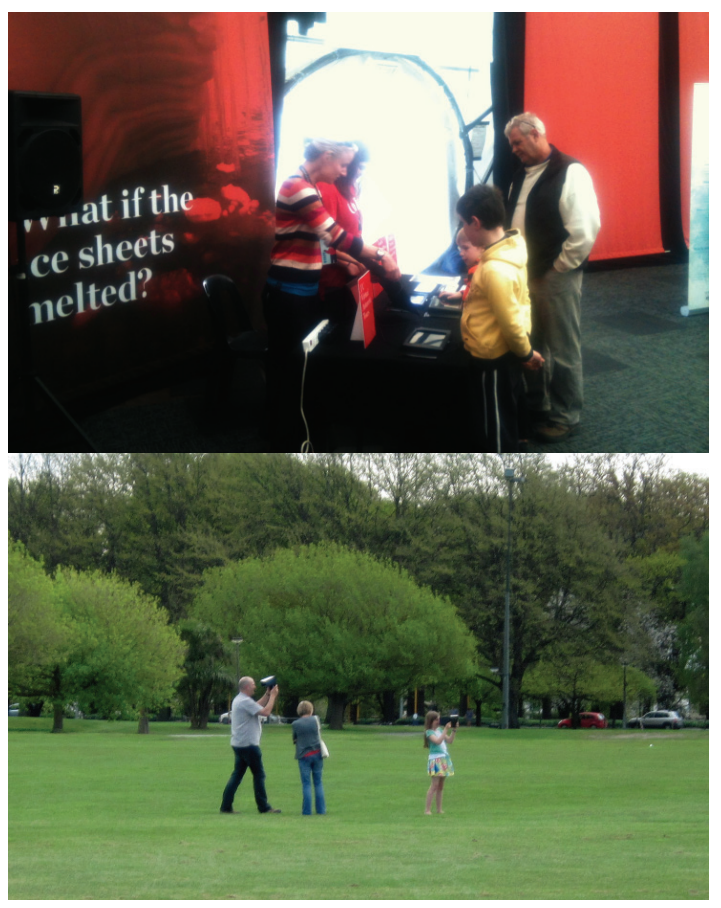

Figure 12: Public demonstration at the NZ IceFest festival - indoor demonstration booth (above) and outdoor tour (below).

For those who tried the application indoors, there were chairs where they could sit down while they used it. These users were allowed to use the tablet for as long as they wanted.

After they finished using the application, we received feedback through a questionnaire from those who were willing, and also collected usage time logs of the application's features. The feedback and logged information were all gathered anonymously.

\subsubsection{Usability questionnaire}

We have received 59 questionnaires but some had missing data, so some of our analyses are based on a smaller sample set, 50 from outdoor users and 5 from indoor users. The average age of the users was 36 years $(S D=20.33)$, with ages ranging from 7 to 63 years. There were 24 male users and 30 female, and some of the users refused to answer.

Overall the experience and ease of use was rated very high with scores of almost 6 on a 7-point Likert scale $(1=$ not at all; $7=$ extremely). The participants also thought that the application was useful for learning about Antarctica and that it provided a better learning experience compared to using the Internet (both questions were measured on a 7-point Likert scale; see Figure 13).



Figure 13: Results from usability questions (Likert scale: 1 7).

To get a more detailed look at the user experience provided by AntarcticAR we have used the Game Experience Questionnaire (GEQ) [21] that has been developed as a measure of game experience. As in [10] we have slightly adapted the wording of 
some questions by changing direct references to "game experience" with "experience". Table 3 shows the internal consistency of the GEQ subscales $(N=33)$.

Table 3: Reliability of the GEQ subscales

\begin{tabular}{l|c}
\multicolumn{1}{c|}{ Subscale } & Cronbach's $\alpha$ \\
\hline Competence & .76 \\
Sensory and Imaginative Immersion & .83 \\
Flow & .81 \\
Tension/Annoyance & .66 \\
Challenge & .58 \\
Negative Affect & .43 \\
Positive Affect & .91 \\
\hline
\end{tabular}

Three of the subscales do not show satisfactory internal consistency: Tension/Annoyance, Challenge, and Negative Affect. A previous study [10] using this questionnaire to measure user experience with an outdoor AR application only found the Challenge subscale to have low reliability. For our analysis we therefore have to interpret results from the three subscales rather cautiously.

Results indicate that AntarcticAR provided a reasonable user experience with above average ratings for Positive Affect, Immersion, Competence and Flow, and low ratings for Negative Affect and Tension Annoyance (see Figure 14). Also, the users thought that the AntarcticAR was not too challenging.

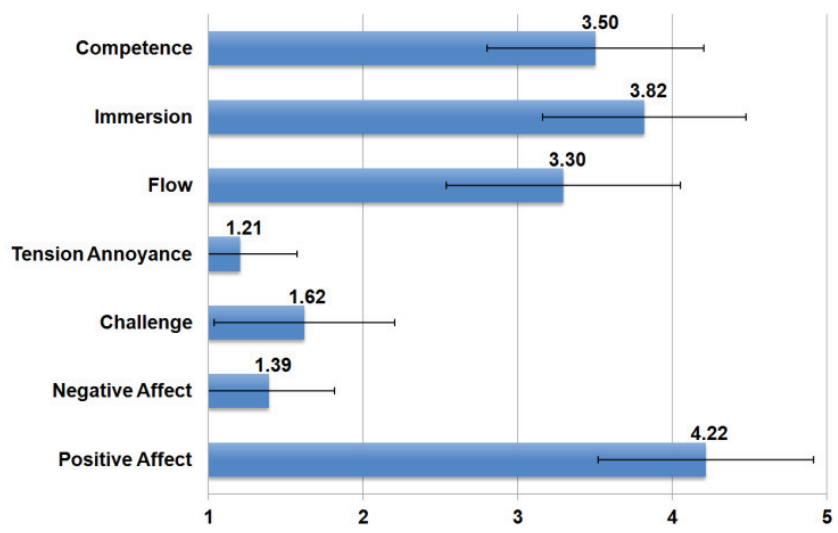

Figure 14: Results from GEQ (Likert scale: 1 5).

We found no significant differences in these subscales between males and females, indoor and outdoor users, or the four different missions. However, when splitting the users by their age median into users younger and older than 40 years, we found that younger users had slightly but statistically significantly higher scores in Competence (young: $M=3.80, S D=.69$; old: $M=3.33, S D=.60$; $U=133.5, p=.03$ ) and Negative Affect (young: $M=1.54$, $S D=.48$; old: $M=1.24, S D=.30 ; U=135, p=.04)$. This indicates that younger users, while feeling more competent, also rated their experience somewhat more negative.

We also asked the users to freely write down what they liked most from the virtual touring experience, what kind of problems they had, and what improvements could be made.

A total of 53 participants answered the question asking their favorite part of the tour. Panorama pictures turned out as the most liked feature, mentioned by 14 users $(26 \%)$, while 11 users replied that watching video clips was their favorite part of the experience $(21 \%)$. Walking in the outdoor environment and following the path was mentioned by 10 users, while another 6 users picked the AR view as their favorite. With these two types of answers combined, 16 users $(30 \%)$ expressed their interest in taking a virtual tour while strolling in an outdoor environment. Then users picked specific content as their favorite, such as penguins, huts, and Scott's diary, while six mentioned they enjoyed the simple user interface with touch screen and map.

When asked if they had any problem during the tour, about $60 \%$ ( 24 out of 40 ) of the participants who answered to this question wrote they had no problem during the experience. The most common problem was taking time to get used to the interface at the beginning (17.5\%; 7 users), and the sunlight causing glare and reflection on the screen even with sun visors on (12.5\%; 5 users). Other problems included software crashing (2 users), starting the tour at the wrong place (1 user), and unusual terms used for explaining content (1 user).

Among 23 participants who answered the question asking what improvements could be made, about half of them (10 users) suggested having more content including pictures and points of interest. Improving the AR visualization of the Antarctic environment was the next major comment (mentioned by 6 people) which included adding more details to the terrain model and hiding real trees in the AR view more completely.

\subsubsection{Usage duration}

From the log files, we identified 188 missions completed by the participants. About two third of the missions $(N=115)$ were completed outdoors on the field, while the others $(N=73)$ were done indoors using touch interaction. We compared the time spent for using different views of the application between these two user groups. While the amount of content was different between the types of missions, we did not take this into account while comparing the two groups since the distribution of the types of mission was similar (see Figure 15). We also note that the results were similar when we only compared 'Visit Ross Island' type of missions.

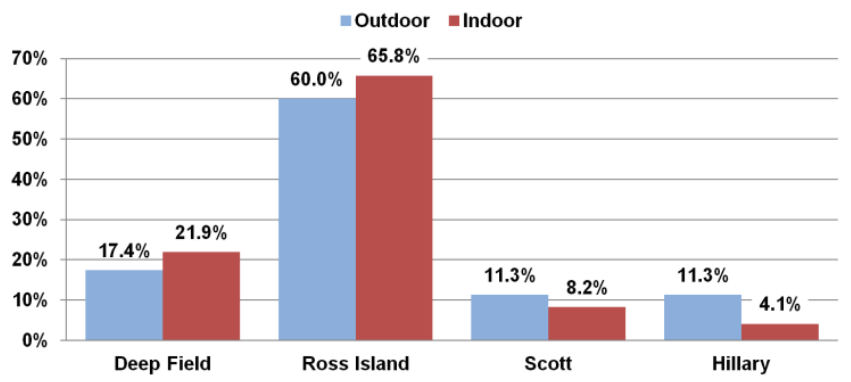

Figure 15: Distribution of the type of completed missions for outdoor and indoor users.

Since the usage duration was not normally distributed, we used non-parametric Mann-Whitney U-tests to analyze the data. Outdoor users spent significantly more time viewing Story content than indoor users $(U=3119, p<.01)$. No significant differences between these groups could be found for the other content types (see Figure 16).

Figure 17 shows the usage duration of navigation views. Indoor participants used the map significantly longer than outdoor users $(U=2002.5, p<.01)$. The AR interface on the other hand was used longer outdoors $(U=55, p<.01)$. The outdoor users spent significantly more time with the AR interface than with the map $(z=-0.08, p<.01)$, while with indoor users this was the other way $(z=4.75, p<.01)$. 




Figure 16: Time spent in content views (in seconds).

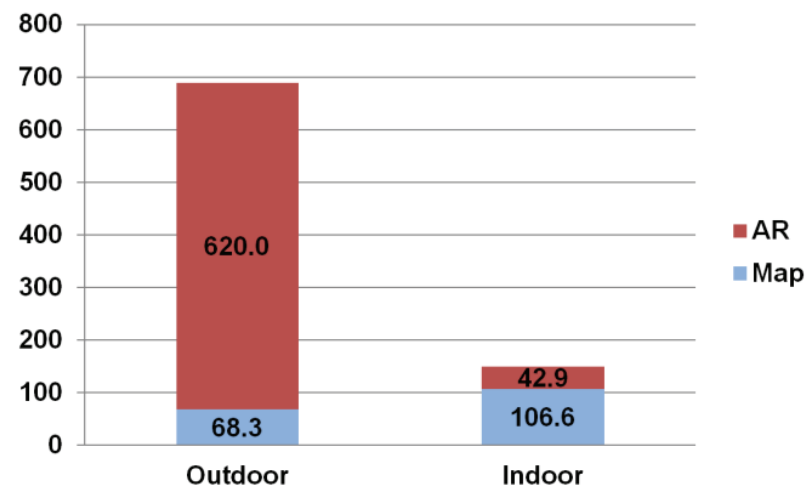

Figure 17: Time spent in navigation views (in seconds).

Among the participants who used the application indoors, about half $(N=38)$ of them did not use the AR interface while the other half $(N=35)$ did. Comparing the overall time for navigation activities between these two groups reveals a significant difference $(U=191, p<.01)$. Interestingly, this difference is only due to the time spent in the AR view, as the time spent for the map is almost exactly the same for both groups (no-AR: $M=106.2$, AR: $M=106.9 ; U=544.5, p=0.183$ ). The ones who used the AR interface spent on average 89.5 seconds using AR. Overall navigation time thus is significantly different between these two groups (see Figure 18).

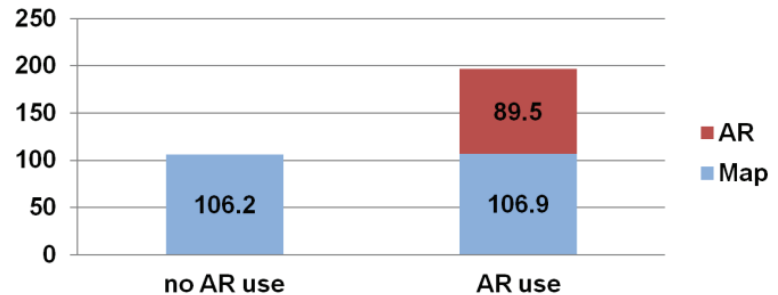

Figure 18: Navigation component use of indoor users (in seconds); split between users who used AR and those who did not.

\subsubsection{Other Observations}

While guiding the participants outdoors, in many cases we observed them enjoying the walking activity itself. Children were especially excited with the outdoor activity and even ran from one place to another from time to time. This also is in line with the questionnaire results in which participants mentioned walking and following the path as one of their favorite parts of the experience.

We also observed participants enjoying the unique experience that the AR view provided. With those who were doing the mission outdoors, once they started using the AR view, they rarely switched back to the Map view. From the log files we found that about $60 \%$ of outdoor participants (70 out of 115 ) never switched back to the map from the AR view, and even those who did mostly used the map view for only brief moments. Some participants, especially children, also enjoyed being in front of each other's camera, trying to put themselves within the virtual scene of Antarctica. We also found that indoor participants also enjoying using the AR view. While most of them were watching the virtual scene and tapping on the flag to get to the next POI, some children even tried to walk out of the booth to get there.

While we had no control over the weather, some of the outdoor users expressed that they enjoyed the cool, cloudy and windy days that made them feel more in an Antarctic environment.

\section{Discussion}

From the results of the user study we can conclude that outdoor AR can be a very promising platform for providing virtual touring experiences. We found that outdoor AR users are more motivated and active in exploring the content provided in the virtual tour. Outdoor users spent more time in reading and watching the content compared to those who used the application indoors. Walking and following the path with the AR view was one of the favorite parts of the experience, besides watching the content in panorama pictures and video clips. Adding to previous work [10], it is interesting that outdoor AR encourages users to walk around and explore more actively even when the real environment has little to do with the virtual environment being explored.

$\mathrm{AR}$ visualization appears to be the favored navigational method for the users in an outdoor environment. While the map provides an exocentric view of the world, AR shows an egocentric view of the environment, making the navigational task more intuitive and easier by providing a first person perspective of the target direction. The results from the user study shows that the users used the AR view significantly more than the map view to navigate in the outdoor environment. Moreover, many outdoor users never returned to the map view once they started using the $A R$ view. The case of children who used the application indoors trying to walk out of the booth shows how intuitive AR is as a navigational guide.

Besides providing an intuitive and easy to use navigational method, AR appears to be contributing more to the overall experience. From the group of participants who used the application indoors, we found the time spent in the AR view was additional to the overall time spent for navigation views, rather than merely replacing the time spent in the map view. This reflects that those who used AR indoors were using it not only as a navigational tool but also as a source of content itself. Indeed, watching the $3 \mathrm{D}$ models such as snowy mountains, huts, tents and penguins was entertaining to both indoor and outdoor users, based on the feedback from users who mentioned this as their favorite part of the experience.

Although the virtual scene had little relationship with the real world, we found that the real environment contributed to the virtual touring experience in various ways. It provided a navigational environment where users can stroll around and make spatial references to the virtual world situated in the real world. As the users walked in the virtual world that was registered to the real space, they felt they were actually touring the virtual scene rather than just watching it on the small screen on the device. The weather and atmosphere of the outdoor environment appeared also to arouse the users' sense of traveling outdoors.

While some of the users pointed out trees in the real world were distracting, we think the background live camera view actually helped users' spatial understanding of the virtual space relative to the real environment. Investigating how much of real world view 
is desirable in a virtual tour application could be an interesting topic for future work. Moreover, applying transitional interfaces [3] that can seamlessly transport users between real and virtual environments could be another interesting path to pursue in further developing outdoor AR virtual touring applications.

Another interesting direction for future research would be investigating how multimodal interaction could contribute to improving the outdoor AR virtual touring experience. While our work mostly focused on providing the visual experience of walking in the Antarctic environment, the application could be enhanced by providing other sensory modalities, such as presenting the howling sound of the wind and vibrating the device during snow storms. In addition, motion and touch gestures could be used to add more interactivity in the tour. For instance, simple motion based game activities at some POIs using metaphorical mapping of real and virtual space [11] could be an interesting component to have in a virtual tour.

We also suggest using a theatrical setup such as providing special costumes or props to the users that could further provoke emotion and participation from them. Learning from artistic performances such as role playing and theatre production could help improve the overall experience.

\section{Conclusion}

In this paper we described the design and implementation of the AntarcticAR mobile outdoor AR application. The application was designed to provide a virtual tour experience to Antarctica. Compared to previous outdoor AR systems, our research shows that outdoor AR can be used for providing virtual tours into spatiotemporally distant spaces that have little connection to the real environment around the user.

We also reported results from a user study showing the feasibility of using outdoor AR technology for virtual tour. The results indicate that the AR view not only worked as a better navigational guide, but also improved the overall user experience by allowing users to experience the virtual scene they were exploring.

For future work, we are looking into improving AR visualization and adding multimodal interaction, and also further investigating the contribution of the real environment in a virtual tour by exploiting transitional interfaces.

\section{REFERENCES}

[1] D. Anguelov, C. Dulong, D. Filip, C. Frueh, S. Lafon, R. Lyon, A. Ogale, L. Vincent, J. Weaver. Google Street View: Capturing the World at Street Level. Computer, vol.43, no.6, pp.32-38, 2010.

[2] C. de Bérigny Wall and X. Wang. Interactive Antarctica: a museum installation based on an augmented reality system. In Proc. of the $3 r d$ international conference on Digital Interactive Media in Entertainment and Arts (DIMEA '08), ACM, New York, NY, USA, pp. 319-325, 2008.

[3] M. Billinghurst, H. Kato, and I. Poupyrev. The MagicBook: a transitional AR interface. Computers \& Graphics 25 (5), pp. 745$753,2001$.

[4] S. E. Chen. QuickTime VR: an image-based approach to virtual environment navigation. In Proc. of the 22nd annual conference on Computer graphics and interactive techniques (SIGGRAPH '95), ACM, New York, NY, USA, pp. 29-38, 1995.

[5] A. D. Cheok, S. W. Fong, K. H. Goh, X. Yang, W. Liu, and F. Farzbiz. Human Pacman: a sensing-based mobile entertainment system with ubiquitous computing and tangible interaction. In Proc. of the 2nd workshop on Network and system support for games (NetGames '03), ACM, New York, NY, USA, pp. 106-117, 2003.

[6] S. Feiner, B. MacIntyre, T. Höllerer, and T. Webster. A touring machine: Prototyping 3D mobile augmented reality systems for exploring the urban environment. In Proc. of the First IEEE
International Symposium on Wearable Computers (ISWC '97), pp. 74-81, 1997.

[7] T. Gleue and P. Dähne. Design and implementation of a mobile device for outdoor augmented reality in the archeoguide project. In Proc. of the 2001 conference on Virtual reality, archeology, and cultural heritage (VAST '01). ACM, New York, NY, USA, pp. 161$168,2001$.

[8] T. Höllerer, S. Feiner, J. Pavlik, Situated Documentaries: Embedding Multimedia Presentations in the Real World, In Proc. of the Third International Symposium on Wearable Computers (ISWC '99), pp. 79-86, 1999

[9] J. Hwang, J. Jung, and G. J. Kim. Hand-held virtual reality: a feasibility study. In Proc. of the ACM symposium on Virtual reality software and technology (VRST '06), ACM, New York, NY, USA, pp. 356-363, 2006.

[10] G. A. Lee, A. Dünser, S. Kim, and M. Billinghurst. CityViewAR: A Mobile Outdoor AR Application for City Visualization. In Proc. of IEEE International Symposium on Mixed and Augmented Reality Arts, Media and Humanities (ISMAR'12), pp.57-64, 2012.

[11] M. Lee and G. J. Kim. Loosely-coupled mixed reality: Using the environment metaphorically. In Proc. of IEEE International Symposium on Mixed and Augmented Reality - Arts, Media and Humanities (ISMAR'09), pp.51-52, 2009.

[12] R. W. Lindeman, G. Lee, L. Beattie, H. Gamper, R. Pathinarupothi, and A. Akhilesh. GeoBoids: A Mobile AR Application for Exergaming. In Proc. of the 11th IEEE International Symposium on Mixed and Augmented Reality - Arts, Media and Humanities (ISMAR2012), Atlanta, GA, USA, pp.93-94, 2012.

[13] P. Milgram and F. Kishino. A taxonomy of mixed reality visual displays. IEICE TRANSACTIONS on Information and Systems 77 (12), pp. 1321-1329, 1994.

[14] M. Mine. Towards virtual reality for the masses: 10 years of research at Disney's VR studio. In Proc. of the workshop on Virtual environments 2003 (EGVE '03), ACM, New York, NY, USA, pp. $11-17,2003$

[15] C. Park, S. C. Ahn, Y. M. Kwon, H. G. Kim, H. Ko and T. Kim. Gyeongju VR theater: a journey into the breath of Sorabol. Presence: Teleoperators \& Virtual Environments, 12(2), pp. 125-139, 2003.

[16] W. Piekarski, D. Hepworth, V. Demczuk, B. Thomas, and B. Gunther. A mobile augmented reality user interface for terrestrial navigation. In Proc. of the 22nd Australasian Computer Science Conference, pp. 122-133, 1999.

[17] H. Rheingold. Virtual Reality. Simon \& Schuster, New York, NY, 1992.

[18] B. Thomas, B. Close, J. Donoghue, J. Squires, P. De Bondi, M. Morris, and W. Piekarski. ARQuake: An outdoor/indoor augmented reality first-person application. In Proc. of the Fourth International Symposium on Wearable Computers (ISWC'00), Atlanta, GA, pp. 139-146, 2000.

[19] V. Vlahakis, N. Ioannidis, J. Karigiannis, M. Tsotros, M. Gounaris, D. Stricker, T. Gleue, P. Daehne, and L. Almeida. Archeoguide: An Augmented Reality Guide for Archaeological Sites. IEEE Computer Graphics and Applications 22 (5), pp. 52-60, 2002.

[20] Climate of Antarctica, Wikipedia. http://en.wikipedia.org/wiki/Climate_of_Antarctica

[21] Game Experience Questionnaire. http://www.gamexplab.nl

[22] Google Earth. http://www.google.com/earth

[23] IMAX. http://www.imax.com

[24] InterANTARCTICA. http://www.interantarctica.com

[25] Junaio. http://www.junaio.com

[26] Layar. http://www.layar.com

[27] Mobile Outdoor AR Framework. http://www.hitlabnz.org/mobileAR

[28] PanoramaGL library. http://code.google.com/p/panoramagl-android

[29] Wikitude. http://www.wikitude.com

[30] You Are Not Here. http://www.youarenothere.org 